\title{
Impacto de la ardilla de vientre rojo sobre la comunidad de aves en la Región Pampeana, Argentina
}

\author{
M. Laura Messetta ${ }^{1}$; Fernando A. Milesi ${ }^{2,3} \&$ M. Laura Guichón ${ }^{\bowtie, 1,3}$ \\ 1. Ecología de Mamíferos Introducidos (EMI), Departamento de Ciencias Básicas, Universidad Nacional de Luján, Buenos \\ Aires, Argentina. 2. Facultad de Ciencias Exactas y Naturales, Universidad de Buenos Aires, Ciudad Universitaria, Buenos \\ Aires, Argentina. 3. Dirección actual: Instituto de Investigaciones en Biodiversidad y Medioambiente (INIBIOMA, UNCo- \\ CONICET), Centro de Ecología Aplicada del Neuquén (CEAN), Junín de los Andes, Neuquén, Argentina
}

\begin{abstract}
Resumen. Las invasiones biológicas son una amenaza para las especies nativas. La ardilla de vientre rojo Callosciurus erythraeus fue introducida en Luján (provincia de Buenos Aires, Argentina) en 1970, donde estableció la principal población silvestre del país. Las ardillas podrían afectar a las aves a través de la depredación de nidadas, de la competencia por alimento y sitios para nidificar, o por interferencia. El objetivo fue comparar la composición, densidad y riqueza de especies de aves en sitios arbolados adentro y afuera del área de distribución de ardillas (680 km² en 2004) en los alrededores de Luján. Se seleccionaron siete pares de sitios (cada par con estructura y composición de la vegetación similares) más otros 14 sitios (sin parear), la mitad en cada condición (con y sin ardillas). En cada uno de los 28 sitios se establecieron 4-5 puntos de conteo de 10-20 m de radio y durante dos días de primavera-verano 2008-2009 se registraron todas las aves vistas/oídas. La composición de las comunidades de aves resultó diferente entre sitios con y sin ardillas, más notoriamente al comparar entre sitios pareados. No se detectaron diferencias en la densidad de aves pero la riqueza de especies fue mayor en los sitios pareados sin ardillas. Se hicieron ensayos de cafetería para evaluar el consumo de huevos por parte de las ardillas y determinar el tamaño de huevo preferido. Ante la oferta de fruta y huevos de tres tamaños, de los 25 ensayos en que las ardillas consumieron fruta, solo en 2 consumieron algún huevo. Nuestros resultados sugieren que las ardillas podrían afectar negativamente a algunas especies de aves pero no a través de la depredación de nidadas.
\end{abstract}

[Palabras clave: ardillas introducidas, Callosciurus erythraeus, densidad de aves, especies invasoras, riqueza de aves]

\begin{abstract}
Aвsтract. Impact of red-bellied squirrels on the bird community of the Pampas Region, Argentina: Biological invasions are a major threat to native species. The red-bellied tree squirrel Callosciurus erythraeus was introduced in Luján, Argentina, in 1970, where it spread rapidly. Squirrels can affect birds through nest predation, interference and competition for food and nesting sites. We aimed to compare community composition, density and richness of birds in forested areas within and outside the range distribution $\left(680 \mathrm{~km}^{2}\right.$ in 2004$)$ of the main population of these squirrels in the country. We selected seven paired sites (each pair had similar vegetation structure and composition) and other 14 sites, half in each condition (with and without squirrels). In each of these 28 sites, we did 4-5 point counts during spring-summer 2008-2009, recording all bird individuals that were seen/heard $<10-20 \mathrm{~m}$ from the observer. Species richness was rarefied to the smallest number of point counts per site. Community composition differed among sites with and without squirrels, particularly for paired sites. We found no differences in bird density though species richness was higher for paired sites without squirrels. To assess if squirrels do consume bird eggs and if they have any egg-size preference, we conducted cafeteria experiments offering fruit and three eggs of different sizes to individual squirrels kept in a large cage $(3 \times 2 \times 2 \mathrm{~m})$ for $<24 \mathrm{~h}$. Few squirrels $(2 / 25)$ consumed the eggs. Our results suggest that squirrels could negatively affect some bird species but not through nest predation.
\end{abstract}

[Keywords: bird density, bird species richness, Callosciurus erythraeus, introduced squirrels, invasive species]

\section{INTRODUCCIÓN}

Durante siglos el hombre ha transportado individuos de una gran variedad de especies fuera de su rango de distribución natural, tanto de manera accidental como intencional. Cierta proporción de las especies introducidas, dependiendo de sus características y de las del nuevo ambiente, superan varias barreras hasta lograr establecerse en condiciones silvestres y expandirse, iniciando una invasión

Editor Asociado: Sergio Lambertucci

$\bowtie$ ardillas@unlu.edu.ar biológica (Blackburn et al. 2011). Muchas de estas especies invasoras tienen impactos negativos sobre el funcionamiento de los ecosistemas y constituyen una amenaza para la biodiversidad (Wittenberg \& Cock 2001; Simberloff et al. 2013). El tipo de impacto y su intensidad es altamente variable y suele estar relacionado con modificaciones en las interacciones de depredación, competencia y mutualismo o con cambios en la estructura de la comunidad y la disponibilidad de 
recursos (Blackburn et al. 2014; Simberloff 2014). Por ejemplo, el aumento en los niveles de depredación y competencia causada por la introducción de mamíferos ha llevado a una marcada disminución, o incluso a la extinción, de numerosas poblaciones de especies nativas (Courchamp et al. 2003; Clout \& Russell 2007; Paolucci et al. 2013).

Varias especies de ardillas fueron introducidas en distintos países, generalmente de manera intencional con fines ornamentales, para ser comercializadas como mascotas o exhibidas en zoológicos (Bertolino 2009). El caso más estudiado fue la introducción de la ardilla gris Sciurus carolinensis en varios países europeos, donde afectó negativamente a las plantaciones forestales y a la ardilla roja europea Sciurus vulgaris por efectos de competencia interespecífica y de transmisión de enfermedades (Gurnell et al. 2004; Rushton et al. 2006; Bertolino et al. 2014). La ardilla gris también podría tener un impacto negativo sobre poblaciones de aves nativas en el Reino Unido (Hewson \& Fuller 2003; Newson et al. 2010), algo que ha sido registrado con la introducción de la ardilla roja Tamiasciurus hudsonicus en regiones de Estados Unidos donde depreda nidadas de aves costeras (Martin \& Joron 2003; Martin et al. 2008). La depredación de huevos o crías de aves es habitual para muchas especies de ardillas dentro de sus áreas de distribución original, como ocurre con varias especies de Norteamérica y Europa (Bayne \& Hobson 2000; Shuttleworth 2001; Willson et al. 2003; Zugmayer \& Koprowski 2007).

La ardilla de vientre rojo Callosciurus erythraeus es la única especie de ardilla introducida en Sudamérica, con poblaciones silvestres solo dentro de Argentina. Esta especie, originaria del sudeste de Asia, también ha sido introducida en Japón, Francia, Bélgica y Holanda (Lurz et al. 2013). En 1970, 10 individuos de esta ardilla arborícola fueron introducidos en una estancia del partido de Luján, provincia de Buenos Aires (Argentina), a partir de los cuales se estableció la primera población silvestre, que continúa en expansión (Aprile \& Chicco 1999; Guichón \& Doncaster 2008). Posteriormente, repetidas traslocaciones y liberaciones de ardillas han resultado en la formación de nuevos focos de invasión en las provincias de Buenos Aires, Córdoba y Santa Fé (Benitez et al. 2013). Las opiniones y actitudes con respecto a la presencia de ardillas introducidas son variadas, desde una percepción negativa que la considera una especie problema que debe ser controlada hasta una percepción positiva que sostiene que es una especie atractiva y vistosa (Borgnia et al. 2013). Los residentes de áreas invadidas por ardillas denuncian daños sobre sus propiedades y actividades productivas e incluso sobre plantas y animales (Guichón et al. 2005). En entrevistas y talleres realizados con residentes (ver Borgnia et al. 2013), varias personas asociaron el incremento en la cantidad de ardillas en sus propiedades con una menor abundancia de aves, incluso reportando ocasionales ataques a nidos. El consumo o destrucción de huevos de aves por parte de la ardilla de vientre rojo fue registrado ocasionalmente en Argentina (Pereira et al. 2003; Zarco 2008) y en Japón (Azuma 1998). Otros posibles efectos de las ardillas sobre las aves son la competencia, ya sea por interferencia o por explotación de recursos como el alimento o los sitios de nidificación, o la modificación del comportamiento o del éxito reproductivo de las aves si su presencia es percibida como una amenaza (Martin 2011), en especial teniendo que en cuenta que en la zona no existen mamíferos nativos diurnos y arborícolas. Estos efectos directos e indirectos no son excluyentes, y más de uno podría afectar la abundancia o diversidad de las aves que habitan las zonas invadidas por ardillas.

El objetivo del presente trabajo fue evaluar el efecto de la presencia de ardillas de vientre rojo sobre la composición, densidad y riqueza de la comunidad de aves en zonas arboladas de la Región Pampeana. Se trabajó bajo las hipótesis de que la presencia de ardillas de vientre rojo afecta negativamente a las aves y que el principal mecanismo es el consumo de sus huevos, por lo que se evaluó experimentalmente si las ardillas consumían huevos cuando estaban disponibles y si había preferencia por un tamaño de huevo en particular, lo que permitiría inferir el grupo de aves más susceptibles. La invasión reciente de las ardillas en un área aún restringida dentro de una región relativamente homogénea permite comparar a las comunidades de aves de sitios arbolados con y sin ardillas suponiendo que serían similares de no ser por su presencia.

\section{MATERIALES y MÉTOdOS}

\section{Área de estudio}

El estudio se realizó en el noreste de la provincia de Buenos Aires, una parte de la región Pampeana, 


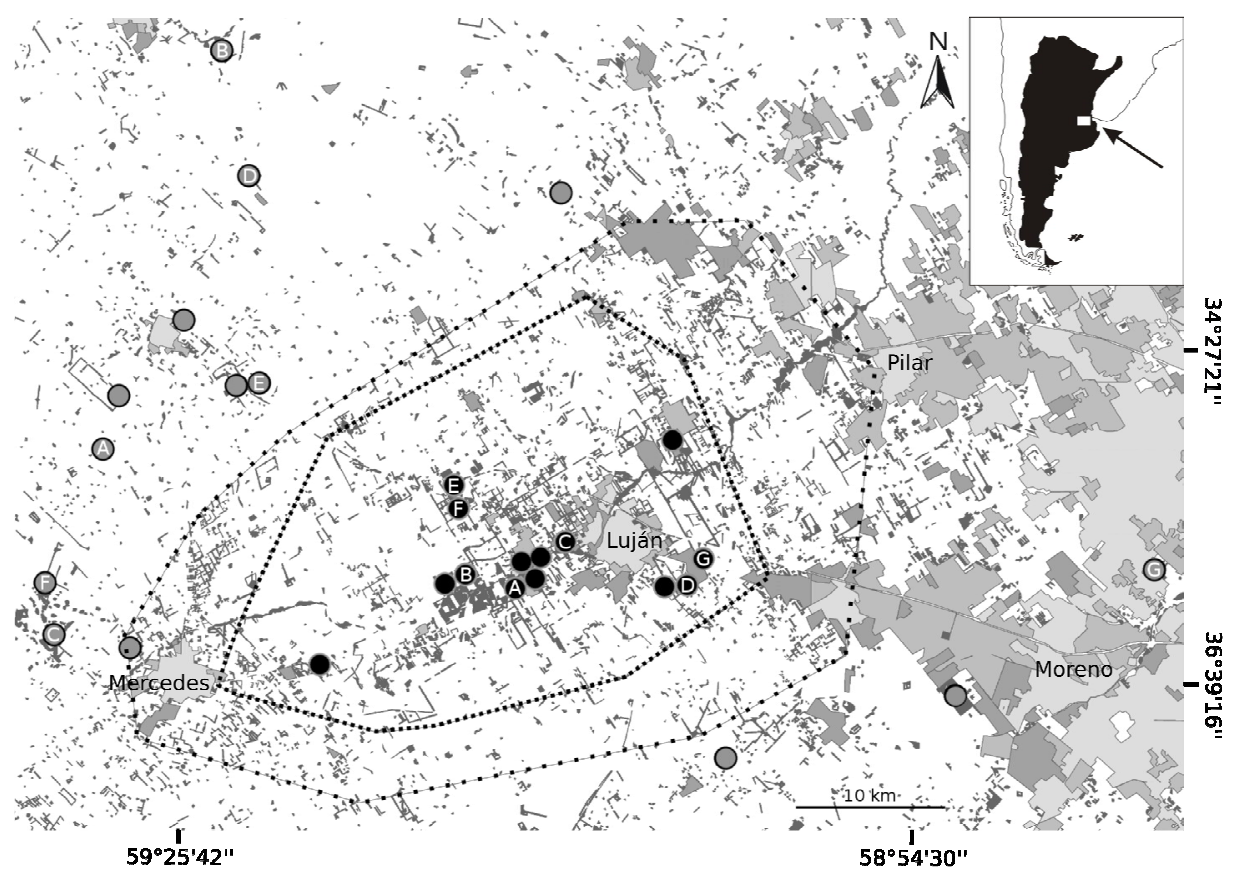

Figura 1. Ubicación de los 28 sitios de muestreo de aves con ardillas de vientre rojo (círculos negros) y sin ardillas (círculos grises) en los alrededores de la ciudad de Luján, provincia de Buenos Aires, Argentina. Los miembros de cada uno de los siete pares de sitios con estructura y composición de la vegetación similares se identifican con una misma letra. Los polígonos indican el área de distribución de la población local de ardillas en 2004 (polígono interno, Guichón et al. 2005) y en 2009 (polígono externo, Benitez et al. 2013). El área presenta hábitats aptos para estas ardillas arborícolas (arboledas en gris oscuro y áreas residenciales, suburbanas y urbanas en tonos de gris de intensidad decreciente) en una matriz de hábitat no adecuado (áreas no arboladas en blanco).

Figure 1. Location of the 28 bird sampling sites with red-bellied squirrels (black circles) and without squirrels (grey circles) in the surroundings of Luján city, province of Buenos Aires, Argentina. See details in Spanish legend.

que originalmente estaba cubierta por pastizales y fue modificada por el desarrollo de actividades agropecuarias y urbanizaciones (Ghersa et al. 1998). Los muestreos se realizaron entre septiembre de 2008 y abril de 2009 en el partido de Luján, donde la ardilla de vientre rojo está presente, y en partidos aledaños (Mercedes, Moreno, San Andrés de Giles y San Antonio de Areco) donde aún no había llegado (Figura 1). Los parches con vegetación arbórea que usan las ardillas en esta región son muy fragmentados, fundamentalmente formados por arboledas ornamentales y para sombra, cortinas de viento, y algunas formaciones naturales y forestaciones comerciales, compuestos en su mayoría por especies introducidas (Ghersa et al. 2002).

\section{Composición, densidad y riqueza de aves}

Se muestrearon 28 sitios arbolados, 14 ubicados dentro del área de distribución de ardillas y 14 fuera de ella. Esa área se estableció en base al área ocupada por ardillas de $680 \mathrm{~km}^{2}$ delimitada en 2004 (Guichón et al. 2005), y comprobando mediante entrevistas y visitas previas que las ardillas aún no habían colonizado los sitios elegidos fuera del área (Figura 1). Para elegir los 28 sitios de muestreo, se identificaron sitios arbolados de al menos 2 ha en base a imágenes satelitales (Google Earth 2008).
Los sitios visitados fueron caracterizados en función de la vegetación arbórea predominante, si era de crecimiento espontáneo o implantado, la cantidad de estratos vegetales (arbóreo, arbustivo y herbáceo) y la altura del estrato herbáceo. Los 28 sitios incluyeron arboledas de distinto tamaño para cada grupo (con y sin ardillas) con vegetación arbórea predominante de una o varias de las siguientes especies: Casuarina spp., Eucalyptus spp., Fraxinus spp., Gleditsia triacanthos, Ligustrum spp., Melia azedarach, Morus spp., Quercus spp., Tipuana spp. Se seleccionaron siete pares de sitios accesibles con la misma especie arbórea dominante y similares estructura y composición de los estratos de vegetación, uno dentro y otro fuera del área con ardillas (siete bloques de sitios pareados). Por ejemplo, un bloque estaba formado por dos sitios con una plantación de Eucalyptus spp., sin estrato arbustivo y con estrato herbáceo corto, un sitio con y otro sin ardillas. Finalmente, se incluyeron muestreos en otros 14 sitios (sin parear) que incorporaban la variabilidad de las arboledas de la región. Los muestreos se realizaron en fechas cercanas en los dos sitios de cada par. De esta manera cualquier posible cambio sistemático en la comunidad de aves con el tiempo afectaría de la misma manera a los muestreos en ambos grupos de sitios (con y sin ardillas), que resultaron temporalmente intercalados a lo largo de la temporada. 
Cada uno de los 28 sitios fue visitado dos veces, en días diferentes, entre las 6:30 y las 10:00 h para realizar los muestreos de aves, evitando días de lluvia o viento fuerte. En cada sitio se ubicaron $4 \mathrm{o}$ 5 puntos de muestreo distanciados 100 m entre sí. En cada punto se registró la cantidad de individuos de cada especie de ave que fueron vistos u oídos dentro de un círculo de $20 \mathrm{~m}$. En aquellos sitios donde la visibilidad horizontal era reducida por la abundante vegetación herbácea-arbustiva se usó un radio fijo de $10 \mathrm{~m}$. El tiempo de muestreo en cada punto fue de ocho minutos, luego de dejar pasar cinco minutos desde la llegada al centro del punto de muestreo, con un tiempo de viaje entre puntos del mismo sitio de 2-5 minutos. Para aquellas especies que fueron oídas y luego vistas en el transcurso del mismo periodo de conteo, se reemplazó el número de individuos oídos por el número de individuos vistos (Marsden et al. 2001).

\section{Análisis de datos}

Se calculó la densidad y la riqueza de especies de aves para cada uno de los 28 sitios relevados. La densidad de aves se calculó suponiendo áreas muestreadas de $20 \mathrm{~m}$ de radio en todos los casos. Para los sitios con puntos de $10 \mathrm{~m}$ de radio se podría estar subestimando la densidad si había individuos (no contados) entre los 10 y $20 \mathrm{~m}$ de radio, pero los diferentes ensayos de extrapolación (e.g., multiplicar por cuatro los individuos registrados) resultaban en valores de densidad muy elevados. Dado que los sitios de 10 y $20 \mathrm{~m}$ de radio estaban balanceados entre tratamientos, esta posible subestimación no afecta la evaluación de las diferencias entre sitios con y sin ardillas. La riqueza se calculó como el número de especies registradas en dos visitas a los 4-5 puntos en cada sitio. Como el esfuerzo de muestreo fue ligeramente distinto entre sitios, la riqueza de especies se rarefaccionó al menor número de visitas (dos visitas a cuatro puntos $=8$ muestras) usando el programa ESTIMATES (Colwell et al. 2004; Colwell 2009). Ninguna de las curvas estimadas alcanzó una asíntota clara, lo que sugiere que la riqueza real en los sitios es mayor y que, si bien se detectaron la mayoría de las especies, el esfuerzo de muestreo realizado solo alcanzó para registrar las aves más frecuentes o conspicuas.

La composición de las comunidades de aves en sitios con y sin ardillas se describió mediante escalamientos multidimensionales no-métricos (NMDS) basados en el índice cuantitativo semimétrico de Bray-Curtis como medida de disimilitud entre sitios. Se intentaron escalamientos en dos dimensiones pero en el caso de todos los sitios se agregó un tercer eje para obtener un valor de stress más razonable (Quinn \& Keough 2002). Además, las comunidades de aves se compararon estadísticamente mediante análisis multivariados de partición de la varianza a partir de matrices de distancia (índice Bray-Curtis), que establece la significación estadística mediante permutaciones de los datos originales (permutational manova: Anderson 2001). La matriz sitios-especies fue doblemente estandarizada (dividiendo las columnas por la abundancia máxima de cada especie y luego las filas por la abundancia total de cada sitio) antes de ambos análisis. Los análisis se hicieron con las funciones metaMDS y adonis del paquete vegan (Oksanen et al. 2013) dentro del sistema estadístico R (R Core Team 2013), siguiendo el esquema de diseño experimental ya descripto: 999 permutaciones libres de los 28 sitios para establecer la distribución esperada del estadístico bajo la hipótesis nula, o 99 permutaciones restringidas (usando el comando strata) dentro de cada uno de los siete pares de sitios para el análisis de los sitios pareados. Las hipótesis estadísticas fueron evaluadas estableciendo $\alpha=0.05$.

Las diferencias en la densidad y en la riqueza de especies de aves entre sitios con y sin ardillas se evaluaron mediante pruebas de $t$ usando los datos de los 28 sitios (14 en zonas con ardillas y 14 sin ardillas) y mediante pruebas de $t$ pareadas usando los datos de los 7 pares de sitios, usando el software INFOSTAT (Infostat 2004). Las dos comparaciones estadísticas intentan sendas aproximaciones para aumentar la potencia estadística: el primer análisis tiene un mayor número de réplicas ( $n=14$ por grupo) pero incluye toda la variabilidad de sitios arbolados de la región; el segundo, disminuye la variabilidad esperada en la variable respuesta comparando sitios estructuralmente similares pero tiene un número de pares de sitios más bajo $(n=7)$.

\section{Consumo de huevos}

Se hicieron ensayos de cafetería en cautiverio para evaluar el consumo de huevos y determinar si hay un tamaño de huevo preferido por las ardillas (Bradley \& Marlzuff 2003). Los ensayos se realizaron entre enero y marzo de 2008 y entre febrero y marzo de 2009, dentro de una jaula de $3 \times 2 \times 2 \mathrm{~m}$ de alambre tejido construida en un sitio arbolado habitualmente utilizado por las ardillas dentro del predio de la Universidad Nacional de Luján. El interior de la jaula contenía árboles $\mathrm{y}$ arbustos, un refugio artificial, y tres nidos naturales de aves (nidos viejos o abandonados recolectados en las cercanías). En cada nido se colocaba un huevo de uno de los tres tamaños a evaluar: grande (huevo de Gallina, Gallus gallus), mediano (huevo de Paloma, Columba livia o de Codorniz, Coturnix coturnix) o pequeño (huevo de Diamante, Taeniopygia sp.), obtenidos de criaderos de aves o del mercado. Además, se ofrecía una porción de fruta (banana, ciruela o naranja) en una rama cercana a los nidos. Se capturaron ardillas usando trampas jaula de captura viva colocadas en la misma arboleda donde se realizaban los ensayos, liberando las ardillas marcadas (parche de pelo cortado) en el sitio de captura luego de cada ensayo. Se realizaron 30 pruebas de cafetería con ardillas diferentes, minimizando el tiempo de cautiverio previo de cada individuo, sin ofrecerle alimento fuera del ensayo para no alterar sus dietas y no modificar de manera desconocida la preferencia por huevos. En cada ensayo se liberó 


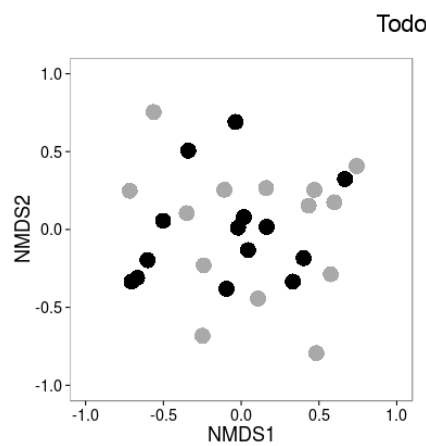

Todos los sitios

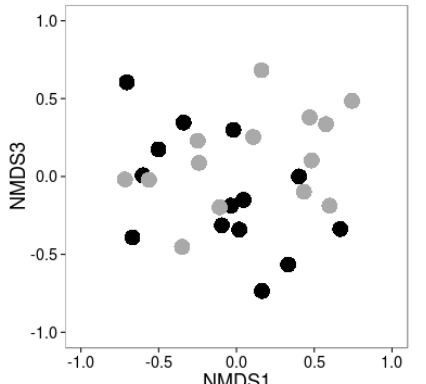

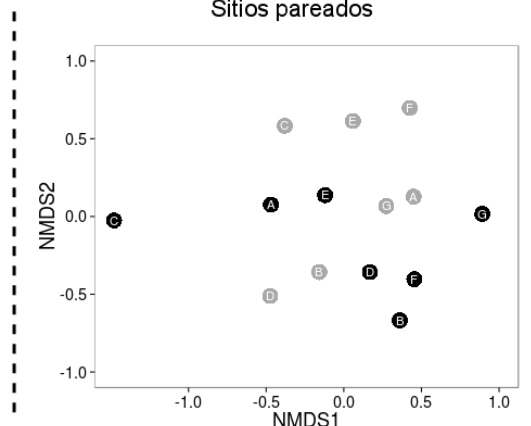

Figura 2. Ordenamiento multidimensional (NMDS) de las comunidades de aves de los sitios relevados con ardillas (negro) y sin ardillas (gris), en tres dimensiones para el análisis de todos los sitios $(n=28)$ y en dos dimensiones para el análisis pareado $(\mathrm{n}=14)$. Los miembros de cada par de sitios con estructura y composición de la vegetación similares se identifican con la misma letra.

Figure 2. Non-metric dimensional scaling (NMDS) of bird communities sampled in sites with squirrels (black) and without squirrels (grey), in three dimensions for the analysis of all sites $(n=28)$ and in two dimensions for the pairedsites analysis $(n=14)$. Paired sites that had similar composition and structure of their vegetation are identified with the same letter.

una ardilla dentro de la jaula y se registró durante no más de $24 \mathrm{~h}$ el consumo, ruptura o desplazamiento de huevos y fruta. Los ensayos se consideraron válidos solo cuando los individuos consumieron algún tipo de alimento, ya sea huevo o fruta, dentro del período de observación, descartando así los individuos que no se dedicaron a explorar y alimentarse.

\section{Resultados}

Se realizaron 244 muestreos de aves (122 puntos visitados dos veces) en los 28 sitios seleccionados. Se registraron 420 individuos pertenecientes al menos a 35 especies de aves en sitios sin ardillas y 311 individuos pertenecientes, al menos, a 27 especies de aves en sitios con ardillas. Considerando solo los 14 sitios pareados, se registraron 264 individuos y 29 especies de aves en sitios sin ardillas y 214 individuos y 22 especies de aves en sitios con ardillas (Tabla 1).

\section{Composición de las comunidades de aves}

La composición de las comunidades de aves de los sitios con y sin ardillas no resultaron equivalentes, aunque la evidencia estadística es más fuerte en el caso de la comparación entre sitios estructuralmente similares $(n=$ 14 , pseudo-F=1.54, $p=0.03$ ) que al comparar las comunidades de aves de todos los sitios muestreados $(\mathrm{n}=28$, pseudo- $\mathrm{F}=1.62, p=0.063)$. En este último análisis que incluye todos los sitios, las comunidades de aves en presencia y ausencia de ardillas se encuentran muy solapadas, con sutiles diferencias de posición sobre todos los ejes de un ordenamiento NMDS en tres dimensiones (stress $=0.166$; Figura 2). En el ordenamiento correspondiente al análisis pareado, en cambio, los sitios con y sin ardillas resultan más dispersos entre sí en sendos ejes de un NMDS bidimensional (stress $=0.151$; Figura 2). Los grupos de sitios con y sin ardillas se encuentran menos solapados debido a diferencias de posición sobre un eje: en la mayoría de los sitios pareados, el sitio sin ardillas tiene un valor igual o mayor al de su par con ardillas sobre el eje 2 (Figura 2).

\section{Densidad y riqueza de aves}

La densidad de aves no difirió significativamente entre sitios con y sin ardillas, tanto al comparar todos los sitios muestreados $(\mathrm{n}=14, \mathrm{t}=-1.30, p=0.10)$ como al analizar solo los sitios pareados $(\mathrm{n}=7, \mathrm{t}=$ $-1.15 p=0.15$ ) (Figura 3). La riqueza de especies de aves entre sitios con y sin ardillas tampoco difirió significativamente al comparar todos los sitios independientemente de su vegetación $(\mathrm{n}=14, \mathrm{t}=-1.02, p=0.16)$. Sin embargo, al

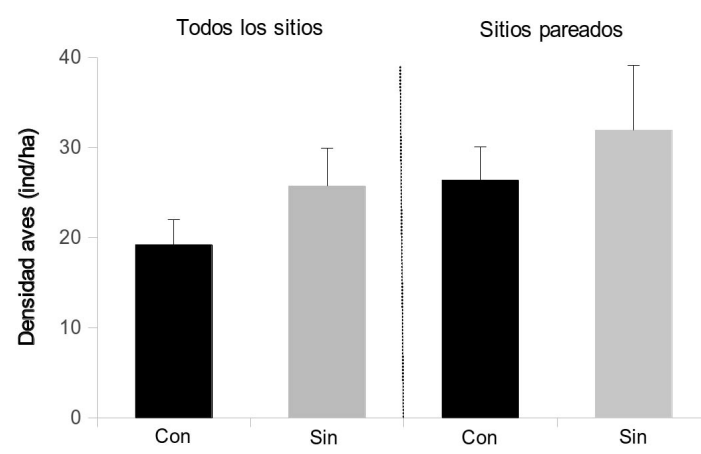

Figura 3. Densidad media de aves ( \pm EE) para todos los sitios muestreados $(n=28)$ y para los sitios pareados $(n=14)$, con ardillas (negro) y sin ardillas (gris).

Figure 3. Mean density of birds $( \pm$ SE) for all sampling sites $(n=28)$ and for paired-sites $(n=14)$, with squirrels (black) and without squirrels (grey). 
Tabla 1. Densidad media (ind/ha) de cada una de las especies de aves registradas en los muestreos realizados en todos los sitios $(n=28)$ y en los sitios pareados $(n=14)$, con y sin presencia de ardillas. Se indica el tamaño de huevo $(T H)$ de cada especie, categorizado en grande $(\mathrm{G})$, mediano $(\mathrm{M})$ y pequeño $(\mathrm{P})$ según el tamaño corporal de los adultos (en base a datos en Narosky \& Yzurieta 2003).

Table 1. Mean density (ind / ha) of each bird species recorded throughout the study in all sampling sites $(\mathrm{n}=28)$ and in paired sites $(n=14)$, with and without squirrels. We indicate the egg size of each species, categorised in large $(G)$, medium $(\mathrm{M})$ and small $(\mathrm{P})$ according to body size of adults (based on Narozky \& Yzurieta 2003).

\begin{tabular}{|c|c|c|c|c|c|}
\hline \multirow[b]{3}{*}{ Especie } & \multirow[b]{3}{*}{$\mathrm{TH}$} & \multicolumn{2}{|c|}{ Todos los sitios } & \multicolumn{2}{|c|}{ Sitios pareados } \\
\hline & & Ardillas & Sin Ard & Ardillas & Sin Ard \\
\hline & & Aves (ind/ha) & Aves (ind/ha) & Aves (ind/ha) & Aves (ind/ha) \\
\hline Syrigma sibilatrix (Chiflón) & G & - & 0.06 & - & 0.11 \\
\hline Buteo magnirostris (Taguató común) & G & 0.20 & 0.07 & - & - \\
\hline Milvago chimango (Chimango) & G & 0.70 & 0.43 & 1.39 & 0.14 \\
\hline Columba picazuro (Paloma picazuró) & G & 1.45 & 0.87 & 1.82 & 0.37 \\
\hline Columba maculosa (Paloma manchada) & G & - & 0.43 & - & - \\
\hline Columba livia (Paloma doméstica) & G & 0.47 & 0.74 & 0.94 & 1.48 \\
\hline Zenaida auriculata (Torcaza) & $\mathrm{M}$ & 0.50 & 0.70 & 0.74 & 1.25 \\
\hline Columbina picui (Torcacita común) & $\mathrm{M}$ & 0.18 & 0.11 & 0.23 & 0.23 \\
\hline Leptotila verreauxi (Yerutí) & $\mathrm{M}$ & 0.64 & 0.51 & 0.60 & 0.91 \\
\hline Myiopsitta monachus (Cotorra) & $\mathrm{M}$ & - & 0.20 & - & 0.40 \\
\hline Chlorostilbon aureoventris (Picaflor común) & $\mathrm{P}$ & 0.14 & - & 0.14 & - \\
\hline Colaptes melanochloros (Carpintero real común) & $\mathrm{M}$ & 0.41 & 0.31 & - & - \\
\hline Picoides mixtus (Carpintero bataraz chico) & $\mathrm{M}$ & - & 0.06 & - & 0.11 \\
\hline Lepidocolaptes angustirostris (Chinchero chico) & $\mathrm{M}$ & 0.44 & 1.32 & 0.34 & 1.11 \\
\hline Furnarius rufus (Hornero) & $\mathrm{M}$ & 2.02 & 2.84 & 2.96 & 3.70 \\
\hline Synallaxis frontalis (Pijuí frente gris) & $\mathrm{M}$ & 0.13 & 0.07 & 0.11 & - \\
\hline Pachyramphus polychopterus (Anambé común) & $\mathrm{M}$ & 0.26 & 0.60 & 0.23 & 0.26 \\
\hline Myiodynastes maculatus (Benteveo rayado) & $\mathrm{M}$ & 0.43 & 0.31 & 0.45 & 0.23 \\
\hline Machetornis rixosa (Picabuey) & $\mathrm{M}$ & 0.07 & 0.07 & - & - \\
\hline Pitangus sulphuratus (Benteveo común) & $\mathrm{M}$ & 1.91 & 1.86 & 2.87 & 2.90 \\
\hline Tyrannus melancholicus (Suirirí real) & $\mathrm{M}$ & 0.11 & 0.57 & 0.23 & 0.77 \\
\hline Tyrannus savana (Tijereta) & $\mathrm{M}$ & - & 0.17 & - & 0.34 \\
\hline Suiriri suiriri (Suirirí común) & $\mathrm{M}$ & 0.06 & - & - & - \\
\hline Serpophaga subcristata (Piojito común) & $\mathrm{P}$ & - & 0.13 & - & 0.11 \\
\hline Troglodytes musculus (Ratona común) & $\mathrm{P}$ & 1.81 & 2.97 & 2.56 & 4.52 \\
\hline Turdus amaurochalinus (Zorzal chalchalero) & $\mathrm{M}$ & 0.27 & 0.40 & 0.40 & 0.80 \\
\hline Turdus rufiventris (Zorzal colorado) & $\mathrm{M}$ & 1.81 & 3.10 & 3.07 & 5.09 \\
\hline Polioptila dumicola (Tacuarita azul) & $\mathrm{P}$ & 0.20 & 0.14 & 0.14 & 0.28 \\
\hline Vireo olivaceus (Chiví común) & $\mathrm{P}$ & 0.07 & - & - & - \\
\hline Parula pitiayumi (Pitiayumí) & $\mathrm{P}$ & 0.11 & 0.27 & 0.11 & 0.11 \\
\hline Piranga flava (Fueguero común) & $\mathrm{M}$ & - & 0.28 & - & - \\
\hline Sicalis luteola (Misto) & $\mathrm{P}$ & - & 0.06 & - & 0.11 \\
\hline Sicalis flaveola (Jilguero dorado) & $\mathrm{P}$ & 0.18 & 1.29 & 0.23 & 1.17 \\
\hline Zonotrichia capensis (Chingolo) & $\mathrm{P}$ & 3.51 & 1.15 & 5.94 & 1.65 \\
\hline Carduelis magellanica (Cabecitanegra común) & $\mathrm{P}$ & - & 0.13 & - & 0.11 \\
\hline Molothrus bonariensis (Tordo renegrido) & $\mathrm{M}$ & - & 0.06 & - & 0.11 \\
\hline Agelaioides badius (Tordo músico) & $\mathrm{M}$ & 0.27 & 0.94 & 0.26 & 1.17 \\
\hline Passer domesticus (Gorrión) & $\mathrm{M}$ & - & 0.47 & - & 0.65 \\
\hline No identificados & & 0.88 & 2.05 & 0.63 & 1.73 \\
\hline
\end{tabular}

comparar los sitios pareados se detectó una mayor riqueza de especies promedio en los sitios a los que las ardillas aún no llegaron $(\mathrm{n}=7, \mathrm{t}=-2.09, \mathrm{p}=0.04)$ (Figura 4). Once especies de aves se detectaron exclusivamente en sitios sin ardillas y tres especies solo en sitios con ardillas (Tabla 1).

\section{Consumo de huevos}

Las ardillas consumieron huevo o fruta en 25 de los 30 ensayos realizados. En todos los casos los individuos empezaron consumiendo la fruta. En general las ardillas no mostraron particular interés por los huevos: solo 6 individuos (24\%) los manipularon. Algunos 


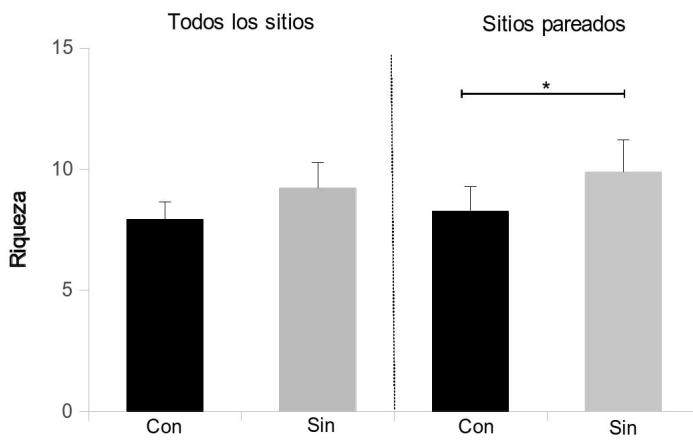

Figura 4. Riqueza de especies de aves ( \pm EE) para todos los sitios muestreados $(n=28)$ y para los sitios pareados $(n=14)$, con ardillas (negro) y sin ardillas (gris). *: diferencias significativas entre condiciones $(p<0.05)$.

Figure 4. Bird species richness $( \pm \mathrm{SE})$ for all sampling sites $(n=28)$ and for paired-sites $(n=14)$, with squirrels (black) and without squirrels (grey). *: significant differences between conditions $(\mathrm{p}<0.05)$.

perforaron la cáscara, dejaron marcas de dientes o rompieron el huevo pero sin consumir su contenido $(n=4)$. Solo 2 ardillas ( $8 \%$ de los 25 ensayos válidos) consumieron el contenido del huevo. Los huevos consumidos o rotos siempre fueron pequeños o medianos; en ningún caso las ardillas consumieron o dejaron marcas sobre los huevos grandes.

\section{DISCUSIÓN}

Este es el primer estudio que evalúa el impacto de la ardilla de vientre rojo sobre la avifauna de la Región Pampeana argentina, donde fue introducida en la década de 1970. Los resultados indican una composición diferente de la comunidad de aves y una menor riqueza de aves en los sitios invadidos por ardillas, aunque solo en comparaciones que controlan la variabilidad estructural y de tipo de vegetación dominante de los sitios (árboles exóticos implantados o espontáneos). Al considerar mayor variabilidad entre sitios, la densidad y la riqueza de aves en sitios con ardillas mostraron menores valores promedio que los de sitios sin ardillas (e.g. densidad $20-30 \%$ mayor en sitios sin ardillas) pero no encontramos evidencia estadística de una diferencia sistemática entre tratamientos. Esto sugiere efectos globales de poca magnitud o, más probablemente, es reflejo de la alta variabilidad espacio-temporal de la comunidad de aves en los sitios con y sin ardillas, lo que sugiere la existencia de otros factores ambientales que resultan más determinantes. Dadas las características de nuestros muestreos, estos resultados deben interpretarse como referidos a las especies más comunes o conspicuas de aves y considerarse con mayor cautela para las especies de aves raras. Efectos de poca magnitud especieespecíficos podrían detectarse con un trabajo de campo aún más intenso, aumentando el número de sitios relevados y haciendo especial hincapié sobre las especies de aves raras o poco abundantes en esta zona, cuyas poblaciones podrían ser más susceptibles a efectos negativos dados por la presencia de ardillas. La hipótesis de una respuesta parcial o especie-específica es consistente con el cambio de composición observado al comparar pares de sitios estructuralmente similares con y sin ardillas.

El efecto de la introducción de especies sobre la riqueza de aves nativas ha sido explicado fundamentalmente por efectos directos como la depredación (Roff \& Roff 2003; Caut et al. 2008) o mediante efectos indirectos como la modificación de la estructura de la vegetación (Zalba \& Cozzani 2003). En otras regiones se ha observado una menor abundancia de aves asociada a la presencia de ardillas por efectos directos, como la depredación de huevos y crías por la ardilla roja (Tamiasciurus hudsonicus) tanto en zonas donde es nativa como en zonas donde ha sido introducida (Bayne \& Hobson 2002; Martin \& Joron 2003; Siepielsky 2006; Martin et al. 2008), por la ardilla roja de Graham (Tamiasciurus hudsonicus grahamensis; Zugmayer \& Koprowski 2007), y por la ardilla roja europea (Sciurus vulgaris; Shuttleworth 2001).Lomismoseha observadoen condiciones experimentales en cautiverio, por la ardilla voladora del norte (Glaucomys sabrinus; Bradley \& Marlzuff 2003). En contraste, en nuestros experimentos de cafetería las ardillas de vientre rojo prefirieron la fruta en todos los casos y solo consumieron el contenido de los huevos en dos oportunidades; los cuatro eventos en que dejaron marcas de dientes, un orificio o destruyeron completamente los huevos pueden ser producto más bien de la actividad exploratoria o destrucción accidental. En estudios experimentales en condiciones naturales llevados a cabo con otra especie de ardilla (Spermophilus richardsonii) también se observaron solo algunos intentos de romper la cáscara de huevos pero escaso o nulo consumo de huevos de aves a menos que estuviesen previamente dañados (Michener 2005). Si bien existen reportes de depredación de nidos por parte de la ardilla de vientre rojo en Argentina (Pereira et al. 2003; Zarco 2008) y en Japón (Azuma 1998), siempre se trata de observaciones aisladas y asistemáticas. Así, la evidencia disponible en Argentina sugiere que la ardilla de vientre rojo no se alimenta 
de huevos habitualmente, como lo indican estudios de dieta que muestran a los frutos, las semillas y las hojas como sus principales alimentos (Zarco et al. 2007; Zarco 2008). La falta de diferencias marcadas en la densidad de aves entre sitios con y sin ardillas de vientre rojo podría deberse a la baja incidencia adicional que tienen las ardillas sobre la tasa de depredación de nidadas de las aves, que suele ser muy alta de todos modos (Ricklefs 1969; Martin 1993). En cualquier caso, la baja frecuencia de consumo de huevos que puede inferirse de nuestros experimentos sugiere que, de haber impactos negativos, la depredación de huevos no sería el principal mecanismo por el cual disminuye la riqueza de aves. Sin embargo, sería conveniente evaluar el posible rol depredador de las ardillas en condiciones naturales mediante seguimiento de nidos. Además, no se puede descartar que especies de características particulares puedan ver afectado su éxito reproductivo por otros impactos como competencia por sitios de nidificación (las ardillas construyen sus nidos en las partes altas de los árboles), interacciones agonísticas o un incremento en la percepción de riesgo de depredación de sus nidadas (Martin 2011).

En síntesis, nuestros resultados no son concluyentes respecto a la relevancia del efecto de las ardillas sobre las aves. Las diferencias observadas en la composición y la riqueza de aves en sitios estructuralmente similares sugieren un efecto negativo de la presencia de ardillas. Si bien la depredación de nidos no sería un mecanismo importante, nueve de las once especies que se observaron únicamente en sitios sin ardillas ponen huevos de tamaño pequeño o mediano, aquellos que parecen susceptibles a la acción intencional o accidental de las ardillas. Dada la dificultad de detectar los efectos más sutiles y de largo plazo de las especies invasoras (Simberloff 2014), nuestros resultados sostienen la importancia de evaluar el impacto que podría tener la ardilla de vientre rojo, una especie invasora reciente en notoria expansión, sobre ciertas especies de aves, particularmente especies raras que son potencialmente más sensibles. Es importante destacar que los muestreos se realizaron en el partido de Luján y otros partidos aledaños, donde existe un alto grado de modificación y perturbación históricas del ambiente por actividades antrópicas. Las especies de aves que ocupan esta región en la actualidad son aquellas que fueron capaces de tolerar o se vieron beneficiadas por el reemplazo de las comunidades naturales por ambientes rurales y urbanos. La inminente invasión de ardillas de vientre rojo en áreas con mayor grado y valor de conservación, como la Reserva Natural Otamendi y el Delta del Río Paraná, puede plantear un escenario notoriamente diferente para especies susceptibles a la presencia de un mamífero exótico arbóreo y diurno, sin equivalentes ecológicos locales.

Agradecimientos. D Acosta, S Almada Chavez, N Mufato y L Miranda colaboraron en los muestreos de campo y pruebas de cafetería; G. Cassini y M. Nardelli aportaron material para los ensayos; V Benitez y C Gozzi ayudaron en distintas etapas del estudio. Dos revisores anónimos contribuyeron a mejorar la versión final del manuscrito. El estudio fue financiado por un subsidio de la Universidad Nacional de Luján, FONCyT (ANPCyTPICT 32195) y Small Ecological Project Grant (British Ecological Society).

\section{BiBLIOGRAFÍA}

ANDERSON, MJ. 2001. A new method for non-parametric multivariate analysis of variance. Austrl Ecol., 26:32-46.

ApriLe, G \& D Chicco. 1999. Nueva especie exótica de mamífero en la Argentina: la ardilla de vientre rojo (Callosciurus erythraeus). Mastozoología Neotropical, 6:7-14.

Azuma, Y. 1998. Nest predation of Japanese White-eye by a Formosan squirrel. Strix, 16:175-176.

BAYNE, EM \& KA HoBSON. 2000. Relative use of contiguous and fragmented boreal forest by red squirrels (Tamiasciurus hudsonicus). Can. J. Zool., 78: 356-365.

BAYNE, EM \& KA HoBSON. 2002. Effects of Red Squirrel (Tamiasciurus hudsonicus) removal on survival of artificial songbird nests in Boreal forest fragments. Am. Midl. Nat., 147:72-79.

Benitez, VV; S Almada Chavez; AC Gozzi; ML Messetta \& ML GuICHÓN. 2013. Invasion status of Asiatic red-bellied squirrels in Argentina. Mamm. Biol., 78:164-170.

Bertolino, S. 2009. Animal trade and non-indigenous species introduction: the world-wide spread of squirrels. Diversity Distrib., 15:701-708.

Bertolino, S; N Cordero di Montezemolo; DG Preatoni; LA WAUTERS \& A MARTINOLI. 2014. A grey future for Europe: Sciurus carolinensis is replacing native red squirrels in Italy. Biol. Invasions, 16:53-62.

BlackBURN, TM; P PYSEK; S BACHER; JT CARLTON; RP DUNCAN; V JAROsIK; JRU Wilson \& DM Richardson. 2011. A proposed unified framework for biological invasions. TREE, 26:333-339.

Blackburn, TM; F Essl; T Evans, PE Hulme; JM JeschKe; ET AL. 2014. A Unified Classification of Alien Species Based on the Magnitude of their Environmental Impacts. PLoS Biol 12: e1001850.

Borgnia, M; V Benitez; C Gozzi \& ML Guichón. 2013. La ardilla de vientre rojo en Argentina y el manejo de especies introducidas como un problema biológico y social. Ecología Austral, 23:147-155.

BradLeY, JE \& JM MARZLUfF. 2003. Rodents as nest predators: influences on predatory behavior and consequences to nesting birds. Auk, 120:1180-1187.

Caut, S; E Angulo \& F Courchamp. 2008. Dietary shift of an invasive predator: rats, seabirds and sea turtles. $J$. Appl. Ecol., 45:428-437.

Clout, MN \& JC Russell. 2007. The invasion ecology of mammals: a global perspective. Wildlife Res., 35: 
180-184.

CoLweLL, RK. 2009. EstimateS: Statistical estimation of species richness and shared species from samples. Version 8.2. http: //purl.oclc.org/estimates

Colwell, RK; XM Chang \& C Jing. 2004. Interpolating, extrapolating and comparing incidence-based species accumulation curves. Ecology, 85:2717-2727.

Courchamp, F; JL Chapuis \& M Pascal. 2003. Mammal invaders on islands: impact, control and control impact. Biol. Rev., 78:347-383.

GHersa, CM; E de La Fuente; S SuARez \& RJC LeON. 2002. Woody species invasion in the Rolling Pampa grasslands, Argentina. Agric. Ecosyst. Environ., 88:271-278.

GHeRsa, CM; MA MartíneZ-GHeRSA \& RJC LEÓN. 1998. Cambios en el paisaje pampeano. Su efecto sobre los sistemas de soporte de la vida. Pp. 38-71 en: Solbrig OT \& L Vainesman (ed.). Hacia una agricultura productiva y sostenible en la pampa argentina. Una visión general prospectiva interdisciplinaria. Editorial CPIA, Argentina.

GOOGLE EARTH. 2008. Imagen satelital de Luján y partidos aledaños, provincia de Buenos Aires, Argentina. http: / / earth.google.com, consultado en febrero de 2008.

Guichón, ML; M Bello \& L Fasola. 2005. Expansión poblacional de una especie introducida en la Argentina: la ardilla vientre rojo Callosciurus erythraeus. Mastozoología Neotropical, 12:189-197.

GUICHÓN, ML \& P DONCASTER. 2008. Invasion dynamics of an introduced squirrel in Argentina. Ecography, 31:211-220.

Gurnell, J; LA Wauters; PWW Lurz \& G Tosi. 2004. Alien species and interspecific competition: effects of introduced eastern grey squirrels on red squirrel population dynamics. J. Anim. Ecol., 73:26-35.

Hewson, CM \& RJ FulLer. 2003. Impacts of Grey Squirrels on woodland birds: an important predator of eggs and young? BTO Research Report No. 328, British Trust for Ornithology, $30 \mathrm{pp}$.

INFOStAT. 2004. InfoStat versión 2004. Grupo InfoStat, FCA, Universidad Nacional de Córdoba, Argentina.

Lurz, PWW; V Hayssen; K Geissler \& S Bertolino. 2013. Callosciurus erythraeus (Rodentia: Sciuridae). Mammalian Species, 48:60-74.

Marsden, S; M Whiffin \& M Galetti. 2001. Bird diversity and abundance in forest fragments and Eucalyptus plantations around an Atlantic forest reserve, Brazil. Biodivers. Conserv., 10:737-751.

Martin, JL; S Allombert \& AJ Gaston. 2008. The effects of deer and squirrels on forest birds: community structure, population density and reproduction. Pp. 93-99 en: Lessons from the islands: introduced species and what they tell us about how ecosystems work. AJ Gaston; TE Golumbia; JL Martin \& ST Sharpe (eds). Environment Canada, Ottawa.

MARTIN, JL \& M JorOn. 2003. Nest predation in forest birds: influence of predator type and predator's habitat quality. Oikos, 102:641-653.

MARTIN, TE. 1993. Nest predation and nest sites: new perspectives on old patterns. BioScience, 43:523-532.

Martin, TE. 2011. The cost of fear. Science, 34:1353-1354.

Michener, GR. 2005. Limits on egg predation by Richardson's ground squirrels. Can. J. Zool., 83: 1030-1037.

NAROSKY, T \& D YZURIETA. 2003. Guía para la identificación de las aves de Argentina y Uruguay. Edición de Oro. Vázquez Mazzini Editores, Buenos Aires.

Newson, SE; EA Rexstad; SR BaIllie; ST BuckLand \& NJ AEBISCHER. 2010. Population change of avian predators and grey squirrels in England: is there evidence for an impact on avian prey populations? J. Appl. Ecol., 47: 244-252.

OKsanen, J; F Guillaume Blanchet; R Kindt; P LegendRe; PR Minchin et AL. 2013. vegan: Community Ecology Package. $\mathrm{R}$ package version 2.0-10. http://CRN.R-project.org/ package $=$ vegan

Paolucci, EA; HJ MacIsaac \& A Ricciardi. 2013. Origin matters: alien consumers inflict greater damage on prey populations than do native consumers. Diversity Distrib., 19:988-995.

Pereira, J; E Haene \& M Babarskas. 2003. Mamíferos de la Reserva Natural de Otamendi. Pp. 115-139 en: E Heane \& J Pereira (eds) Temas de Naturaleza y Conservación 3: Fauna de Otamendieds.). Aves Argentinas/AOP, Buenos Aires.

Quinn, GP \& MJ KeOUgh. 2002. Experimental design and data analysis for biologists. Cambridge University Press, New York. 537 pp.

R CORE TEAm. 2013. $R$ : a language and environment for statistical computing (version 3.0.2 for Linux-GNU). R Foundation for Statistical Computing, Vienna, Austria. http://www.R-project.org/

RICKLEFS, RE. 1969. An analysis of nesting mortality in birds. Smithsonian Contributions to Zoology , 9:1-48.

RofF, DA \& RJ Roff. 2003. Of rats and maoris: a novel method for the analysis of patterns of extinction in the New Zealand avifauna before European contact. Evol. Ecol. Res., 5:759-779.

Rushton, SP; PWW Lurz; J Gurnell; P Nettleton; C BRUEMMER ET AL. 2006. Disease threats posed by alien species: the role of a poxvirus in the decline of the native red squirrel in Britain. Epidemiol. Infect., 134:521-533.

Shuttleworth, CM. 2001. Interactions between the red squirrel (Sciurus vulgaris), great tit (Parus major) and jackdaw (Corvus monedula) whilst using nest boxes. J. Zool., 255:269-272.

SIEPIELSKI, AM. 2006. A possible role for red squirrels in structuring breeding bird communities in lodgepole pine forests. Condor, 108:232-238.

Simberloff, D. 2014. Biologial invasions: what's worht fighting and what can be won? Ecol. Eng., 64:112-121.

SimberlofF, D; JL Martin; P Genovesi; V Maris; DA WardLe et al. 2013. Impacts of biological invasions: what's what and the way forward. TREE, 28:58-66.

Willson, MF; TL De Santo \& KE Sieving. 2003. Red squirrels and predation risk to bird nests in northern forests. Can. J. Zool., 81:1202-1208

WITTENBERG, R \& MJW COCK. 2001. Invasive alien species: a toolkit of best prevention and management practices. CAB International, Wallingford, Oxon, UK. 228 pp.

Zalba, SM \& NC CozZANI. 2004. The impact of feral horses on grassland bird communities in Argentina. Anim. Conserv., 7:35-44.

ZARCO, A. 2008. Hábitos alimentarios de la ardilla de vientre rojo (Callosciurus erythraeus) en la localidad de $\mathrm{La}$ Cumbrecita, Córdoba. Tesis de Licenciatura, Facultad de Ciencias Exactas, Físicas y Naturales, Universidad Nacional de Córdoba, Argentina.

Zarco, A; VV Benitez; G Funes \& ML Guichón. 2007. Hábitos alimentarios de la ardilla de vientre rojo introducida en Argentina. XXI Jornadas Argentinas de Mastozoología, pp. 184.

Zugmeyer, CA \& JL Koprowski. 2007. Avian nestling predation by endangered Mount Graham red squirrel. Southwest. Nat., 52:155-157. 